PROCEEDINGS OF THE

AMERICAN MATHEMATICAL SOCIETY

Volume 136, Number 6, June 2008, Pages 2217-2224

S 0002-9939(08)09266-6

Article electronically published on February 7, 2008

\title{
STRONG PERIODICITY OF LINKS AND THE COEFFICIENTS OF THE CONWAY POLYNOMIAL
}

\author{
NAFAA CHBILI \\ (Communicated by Daniel Ruberman)
}

\begin{abstract}
Przytycki and Sokolov proved that a three-manifold admits a semifree action of the finite cyclic group of order $p$ with a circle as the set of fixed points if and only if $M$ is obtained from the three-sphere by surgery along a strongly $p$-periodic link $L$. Moreover, if the quotient three-manifold is an integral homology sphere, then we may assume that $L$ is orbitally separated. This paper studies the behavior of the coefficients of the Conway polynomial of such a link. Namely, we prove that if $L$ is a strongly $p$-periodic orbitally separated link and $p$ is an odd prime, then the coefficient $a_{2 i}(L)$ is congruent to zero modulo $p$ for all $i$ such that $2 i<p-1$.
\end{abstract}

\section{INTRODUCTION}

Strongly periodic links have been introduced by Przytycki and Sokolov 18 as a class of links providing equivariant surgery presentations of cyclic covers branched along knots, i.e., periodic three-manifolds. This equivariant surgery presentation plays a key role in the study of the Casson-Walker-Lescop invariant as well as the quantum invariants of periodic three-manifolds $[3,4,5,6,8$. 8. Let $p \geq 2$ be an integer. A link $L$ in $S^{3}$ is said to be $p$-periodic if there exists a periodic transformation $h$ of order $p$ such that $f i x(h) \cong S^{1}, h(L)=L$ and $f i x(h) \cap L=\emptyset$. By the positive solution of the Smith conjecture [1] we may assume that $h$ is a rotation by a $2 \pi / p$ angle around the $z$-axis. A $p$-periodic link is said to be strongly $p$-periodic if and only if every component of the quotient link $\bar{L}$ has linking number zero modulo $p$ with the axis of the rotation. The link $L$ is said to be orbitally separated (we write OS for short) if the quotient link is algebraically split. Przytycki and Sokolov [18 proved that a three-manifold is periodic with prime period $p$ if and only if $M$ is obtained from $S^{3}$ by surgery along a strongly $p$-periodic link $L$. Moreover, It can be easily seen that if the quotient manifold $\bar{M}$ is an integral homology sphere, then we may assume that $L$ is orbitally separated.

Murasugi [16] established a congruence formula for the Alexander polynomial of periodic knots. Later, different approaches have been used to give alternative

Received by the editors August 31, 2006.

2000 Mathematics Subject Classification. Primary 57M25.

Key words and phrases. Strongly periodic links, equivariant crossing change, Conway polynomial.

The author was supported by a fellowship from the COE program "Constitution of wide-angle mathematical basis focused on knots", Osaka City University.

(C)2008 American Mathematical Society Reverts to public domain 28 years from publication 
proofs to Murasugi's result [2, 7, 10]. In [15] and [19, similar results have been proved for the multi-variable Alexander polynomial of periodic links.

The Conway polynomial is a renormalization of the Alexander polynomial. If $L$ is a link with $n$ components, then the Conway polynomial of $L$ is of the form $\nabla_{L}(z)=$ $z^{n-1}\left(a_{0}(L)+a_{2}(L) z^{2}+\cdots+a_{2 m}(L) z^{2 m}\right)$, where the coefficients $a_{i}(L)$ are integers. We know that the coefficient $a_{0}(L)$ depends only on the linking matrix of $L$. The second coefficient $a_{2}(L)$ is related to the Casson-Walker-Lescop invariant by the global surgery formula introduced by Lescop [13. The study of the coefficients of the Conway polynomial is of interest partially because they are Vassiliev invariants of suitable orders.

Let $p$ be an odd prime and let $L$ be an OS strongly $p$-periodic link. In [5] we proved that the coefficient $a_{2}(L)$ is congruent to zero modulo $p$. Using this fact and Lescop's surgery formula we have been able to prove that the Casson-WalkerLescop invariant of a periodic three-manifold is equal, modulo $p$, to the product of the signature of the surgery presentation by the order of the first homology group over 8 .

The purpose of this paper is to extend the result proved in [5] for the coefficient $a_{2}$ to other coefficients of the Conway polynomial. Here is our Main Theorem

Main theorem. Let $p$ be an odd prime and let $L$ be an $O S$ strongly p-periodic link. Then $a_{2 i}(L)$ is congruent to zero modulo $p$ for all $i$ such that $2 i<p-1$.

The Main Theorem does not hold for $p=2$. A counterexample is given by considering the Hopf link $H$. Obviously, $H$ is an OS strongly 2-periodic link. The Conway polynomial of $H$ is $\nabla_{H}(z)=z$. This means that the coefficient $a_{0}(H)=1$; hence it is not null modulo 2 .

Here is an outline of the paper. Section 2 introduces strongly periodic links and discusses the notion of equivariant crossing changes. Section 3 reviews some properties of the Conway polynomial needed in the sequel. Finally, Section 4 is devoted to the proof of the Main Theorem.

\section{Strongly PERIOdiC LINKS AND EqUiVARIANT CROSSING CHANGES}

In this section, we first define strongly periodic links. Then we introduce some of their properties. We close the section with a list discussing the different types of equivariant crossing changes.

Definition 2.1. Let $p \geq 2$ be an integer. A link $L$ of $S^{3}$ is said to be $p$-periodic if and only if there exists an orientation-preserving auto-diffeomorphism $h$ of $S^{3}$ such that:

1) $f i x(h)$ is homeomorphic to the circle $S^{1}$,

2) the link $L$ is disjoint from $\operatorname{fix}(h)$,

3) $h$ is of order $p$,

4) $h(L)=L$.

If $L$ is periodic we will denote the quotient link by $\bar{L}$.

The standard example of such a diffeomorphism is given as follows. Let us consider $S^{3}$ as the submanifold of $\mathbb{C}^{2}$ defined by $S^{3}=\left\{\left(z_{1}, z_{2}\right) \in \mathbb{C}^{2} ;\left|z_{1}\right|^{2}+\left|z_{2}\right|^{2}=\right.$ $1\}$ and $\varphi_{p}$ the following diffeomorphism:

$$
\begin{aligned}
\varphi_{p}: \quad S^{3} & \longrightarrow S^{3} \\
\left(z_{1}, z_{2}\right) & \longmapsto \\
& \left(e^{\frac{2 i \pi}{p}} z_{1}, z_{2}\right) .
\end{aligned}
$$


The set of fixed points for $\varphi_{p}$ is the circle $\Delta=\left\{\left(0, z_{2}\right) \in \mathbb{C}^{2}:\left|z_{2}\right|^{2}=1\right\}$. If we identify $S^{3}$ with $\mathbb{R}^{3} \cup \infty, \Delta$ may be seen as the standard $z$-axis.

Recall here that if the quotient link $\bar{L}$ is a knot, then the link $L$ may have more than one component. In general, the number of components of $L$ depends on the linking numbers of the components of $\bar{L}$ with the axis of the rotation.

Recently, Przytycki and Sokolov [18] introduced the notion of strongly periodic links as follows.

Definition 2.2. Let $p \geq 2$ be an integer. A $p$-periodic link $L$ is said to be strongly $p$-periodic if and only if one of the following equivalent conditions holds:

(i) The linking number of each component of $\bar{L}$ with the axis $\Delta$ is 0 modulo $p$.

(ii) The group $\mathbb{Z} / p \mathbb{Z}$ acts freely on the set of components of $L$.

(iii) The number of components of $L$ is $p$ times greater than the number of components of $\bar{L}$.

Remark 2.1. According to condition (iii) in the previous definition, a strongly $p$ periodic link $L$ has $p \alpha$ components, where $\alpha$ is the number of components of the quotient link $\bar{L}$. These $p \alpha$ components are divided into $\alpha$ orbits with respect to the free action of $\mathbb{Z} / p \mathbb{Z}$ (condition (i)).

Assume that $\bar{L}=l_{1} \cup \cdots \cup l_{\alpha}$. There is a natural cyclic order on each orbit of components of $L$, namely,

$$
L=l_{1}^{1} \cup \cdots \cup l_{1}^{p} \cup l_{2}^{1} \cup \cdots \cup l_{2}^{p} \cup \cdots \cup l_{\alpha}^{1} \cup \cdots \cup l_{\alpha}^{p}
$$

where $\varphi_{p}\left(l_{i}^{t}\right)=l_{i}^{t+1} \forall 1 \leq t \leq p-1$ and $\varphi_{p}\left(l_{i}^{p}\right)=l_{i}^{1}$ for all $1 \leq i \leq \alpha$.

Definition 2.3. A $\operatorname{link} L$ in the three-sphere is said to be algebraically split if and only if the linking number of any two components of $L$ is null.

Definition 2.4. Let $p \geq 2$ be an integer. A strongly $p$-periodic link is said to be orbitally separated if and only if the quotient link is algebraically split.

Remark 2.2. A three-manifold $M$ is said to be $p$-periodic if the finite cyclic group of order $p$ acts semi-freely on $M$ with a circle as the set of fixed points. By [18] and 20], we know that a three-manifold is $p$-periodic if and only if $M$ is obtained from $S^{3}$ by surgery along a strongly $p$-periodic link. If the quotient manifold is an integral homology sphere, then we may choose the link $L$ to be orbitally separated. This is a consequence of the fact that the quotient manifold may be obtained by surgery along an algebraically split link.

Remark 2.3. For short, we shall use the term OS link to refer to an orbitally separated link. If $L$ is a strongly $p$-periodic OS link, then for all $s$ and $i \neq j$ we have $\sum_{t=1}^{p} l k\left(l_{i}^{s}, l_{j}^{t}\right)=0$.

Definition 2.5. Let $m$ be a positive integer. A $p$-periodic link $L$ is said to be of type $m$ if $L=K_{1} \cup K_{1}^{\prime} \cup \cdots \cup K_{m} \cup K_{m}^{\prime} \cup L^{\prime}$ such that

(i) $K_{i}$ and $K_{i}^{\prime}$ are fixed by $\varphi_{p}$ for all $i$, and $L^{\prime}$ is an OS strongly $p$-periodic link,

(ii) $\operatorname{Lk}\left(K_{i}, \Delta\right) \equiv-\operatorname{Lk}\left(K_{i}^{\prime}, \Delta\right)$ modulo $p$,

(iii) $\operatorname{Lk}\left(\bar{K}_{i}, l\right)=-\operatorname{Lk}\left(\overline{K_{i}^{\prime}}, l\right)$ for all components $l$ of the quotient link $\overline{L^{\prime}}$.

By convention a $p$-periodic link of type 0 is a strongly $p$-periodic OS link. 

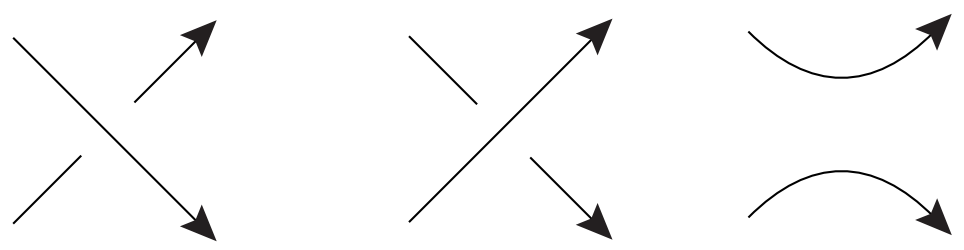

Figure 1

Let $L$ be a $p$-periodic link in the three-sphere. Let $\bar{L}$ be the factor link, so here we have $L=\pi^{-1}(\bar{L})$, where $\pi$ is the canonical surjection corresponding to the action of the rotation on the three-sphere. Let $\bar{L}_{+}, \bar{L}_{-}$and $\bar{L}_{0}$ denote the three links which are identical to $\bar{L}$ except near one crossing where they are as shown in Figure 1. Now, let $L_{p+}:=\pi^{-1}\left(\bar{L}_{+}\right), L_{p-}:=\pi^{-1}\left(\bar{L}_{-}\right)$and $L_{p 0}:=\pi^{-1}\left(\bar{L}_{0}\right)$. We define an equivariant crossing change as a change from $L_{p+}$ to $L_{p-}$ or vice versa. If this crossing change involves two different components of the quotient link, then we call it a mixed equivariant crossing change. Otherwise, it is called a self-equivariant crossing change.

Lemma 2.1. Let $L=K_{1} \cup K_{1}^{\prime} \cup \cdots \cup K_{m} \cup K_{m}^{\prime} \cup L^{\prime}$ be a p-periodic link of type $m$.

1. If we change a crossing between $\bar{K}_{i}$ and $\overline{K_{i}^{\prime}}$, then $L_{p 0}$ has $\left.\sharp L+p-2\right)$ components.

2. If we change a crossing between $\bar{K}_{i}$ or $\overline{K_{i}^{\prime}}$ and $\bar{K}_{j}$ for $i \neq j$, then $L_{p 0}$ has either $(\sharp L+p-2)$ or $(\sharp L-1)$ components.

3. If we change a crossing between $\bar{K}_{i}$ or $\overline{K_{i}^{\prime}}$ and a component of $\overline{L^{\prime}}$, then $L_{p 0}$ is of type $m$ having $(\sharp L-p)$ components.

4. If we change a crossing between two components of $\overline{L^{\prime}}$, then $L_{p 0}$ is of type $m$ having $(\sharp L-p)$ components.

5. If we change a self-crossing in $\overline{L^{\prime}}$, then $L_{p 0}$ is either of type $(m+1)$ having $(\sharp L-p+2)$ components or a periodic link having $(\sharp L+p)$ components.

Proof. The proof is straightforward by analyzing the linking numbers of the components of $\bar{L}_{p 0}$ with the axis of the rotation.

\section{The Conway polynomial}

The Conway polynomial $\nabla$ is an invariant of ambient isotopy of oriented links which can be defined uniquely by the following:

(i) $\nabla_{\bigcirc}(z)=1$

(ii) $\nabla_{L_{+}}(z)-\nabla_{L_{-}}(z)=z \nabla_{L_{0}}(z)$

where $\bigcirc$ is the trivial knot, and $L_{+}, L_{-}$and $L_{0}$ are three oriented links which are identical except near one crossing where they appear as in Figure 1 .

It is well known that if $L$ is a link with $n$ components, then the Conway polynomial of $L$ is of the form $\nabla_{L}(z)=z^{n-1}\left(a_{0}(L)+a_{2}(L) z^{2}+\cdots+a_{2 m}(L) z^{2 m}\right)$, where the coefficients $a_{i}(L)$ are integers.

Now, we shall recall some properties of the Conway polynomial needed in the sequel.

Let $L=l_{1} \cup \cdots \cup l_{n}$ be an oriented $n$-component link in the three-sphere. Let $l_{i j}=\operatorname{Lk}\left(l_{i}, l_{j}\right)$ if $i \neq j$ and define $l_{i i}=-\sum_{j=1, j \neq i}^{n} l_{i j}$. Define the linking matrix $\mathcal{L}$ as $\mathcal{L}=\left(l_{i j}\right)$. The matrix $\mathcal{L}$ is symmetric and each row sums to zero. Hence, every 
cofactor of this matrix is the same. We refer the reader to [9, [11] and [12 for details about the linking matrix.

Theorem $3.1([12])$. Let $L$ be an oriented link with $n$ components. Then $a_{0}(L)=$ $\mathcal{L}_{i j}$, where $\mathcal{L}_{i j}$ is any cofactor of the linking matrix $\mathcal{L}$.

It follows from this theorem that if $L$ is algebraically split, then the first coefficient of the Conway polynomial is zero. Levine [14] proved that the coefficient of $z^{i}$ is null, for all $i \leq 2 n-3$, and he gave an explicit formula for the coefficient of $z^{2 n-2}$.

Proposition $3.2([14])$. If $L$ is algebraically split with $n$ components, then $\nabla_{L}(z)$ is divisible by $z^{2 n-2}$.

\section{Proof of the Main Theorem}

The idea of the proof is to use equivariant crossing changes to reduce strongly periodic OS links to algebraically split links without changing the first coefficients of the Conway polynomial modulo $p$. We first introduce the following lemma, which plays a crucial role in the proof.

Lemma 4.1. Let $p$ be a prime. Then we have the following congruence modulo $p$ :

$$
\nabla_{L_{p+}}(z)-\nabla_{L_{p-}}(z) \equiv z^{p} \nabla_{L_{p 0}}(z)
$$

Proof. See [5].

Remark 4.1. Let $k$ be the number of components of $L_{p+}$. Obviously, the link $L_{p-}$ has $k$ components as well. Let $s$ be the number of components of $L_{p 0}$. According to Lemma 4.1, we have the following congruence modulo $p$ :

$$
\begin{aligned}
& z^{k-1}\left(a_{0}\left(L_{p+}\right)+a_{2}\left(L_{p+}\right) z^{2}+\ldots\right)-z^{k-1}\left(a_{0}\left(L_{p-}\right)+a_{2}\left(L_{p-}\right) z^{2}+\ldots\right) \\
& \quad \equiv z^{p+s-1}\left(a_{0}\left(L_{p 0}\right)+a_{2}\left(L_{p 0}\right) z^{2}+\ldots\right) .
\end{aligned}
$$

Consequently, if $s \geq k-1$, then $a_{2 i}\left(L_{p+}\right) \equiv a_{2 i}\left(L_{p-}\right)$ modulo $p$ for all $i$ such that $2 i<p-1$.

Lemma 4.2. Let $L$ be a p-periodic link of type $m$. If $L$ is algebraically split, then $a_{2 i}(L)$ is congruent to zero modulo $p$ for all $i$ such that $2 i<p-1$.

Proof. We know that $L$ has $2 m+p \alpha$ components. If $\alpha>0$, then the number of components of $L$ is greater than or equal to $p$. Using Levine's theorem we should be able to conclude that $a_{2 i}$ is zero modulo $p$ for all $i$ such that $2 i<p-1$.

If $\alpha=0$, then $L=K_{1} \cup K_{1}^{\prime} \cup \cdots \cup K_{m} \cup K_{m}^{\prime}$. The idea here is to make equivariant crossing changes until getting a split link, without changing the coefficient $a_{2 i}$ modulo $p$. We shall focus on equivariant crossing changes involving $K_{1}$ and the other components. If we change a crossing between $\bar{K}_{1}$ and $\bar{K}_{1}^{\prime}$, then the link $L_{p 0}$ has $2(m-1)+p$ components. However, if we change a crossing between $\bar{K}_{1}$ and $\bar{K}_{j}$ (resp. $\bar{K}_{j}^{\prime}$ ) for $1 \neq j$, then the number of components of $L_{p 0}$ is either $2(m-1)+p$ or $2(m-1)+1$. A simple computation shows that in all cases, Remark 4.1 implies that $a_{2 i}\left(L_{p+}\right)$ is congruent to $a_{2 i}\left(L_{p-}\right)$ modulo $p$ for $2 i<p-1$. It can be easily seen that using these crossing changes we can put $K_{1}$ over the rest of the components of $L$ to get a split link. Since $L$ has the same coefficient $a_{2 i}$ modulo $p$ as a split link, then $a_{2 i}(L)$ is null modulo $p$ for all $i$ such that $2 i<p-1$. This ends the proof. 
Lemma 4.3. If $L$ is of type $m>0$, then $a_{0}(L)$ is null modulo $p$.

Proof. The coefficient $a_{0}(L)$ is computed using the Hoste formula. Recall that $L$ can be written as follows:

$$
L=K_{1} \cup K_{1}^{\prime} \cup \cdots \cup K_{m} \cup K_{m}^{\prime} \cup l_{1}^{1} \cup \cdots \cup l_{1}^{p} \cup \cdots \cup l_{\alpha}^{1} \cup \cdots \cup l_{\alpha}^{p} .
$$

It is easy to see that the linking number of any couple of two invariant components of $L$ is null modulo $p$. Moreover for such a component $K$ we have:

$$
L k\left(K, l_{j}^{1}\right)=L k\left(K, l_{j}^{2}\right)=\cdots=L k\left(K, l_{j}^{p}\right) \quad \forall 1 \leq j \leq \alpha .
$$

By Definition 2.5, (iii) we have $\operatorname{Lk}\left(\bar{K}_{i}, l\right)=-\operatorname{Lk}\left(\overline{K_{i}^{\prime}}, l\right)$ for all components $l$ of the quotient link $\overline{L^{\prime}}$, where $L^{\prime}$ is the strongly periodic part of $L$. Thus, $\left.\sum_{t=1}^{p} \operatorname{Lk}\left(K_{i}, l_{j}^{t}\right)\right)$ $\left.=-\sum_{t=1}^{p} \operatorname{Lk}\left(K_{i}^{\prime}, l_{j}^{t}\right)\right)$ for all $1 \leq i \leq m$ and $1 \leq j \leq \alpha$. Notice that in each of the two summations above the $p$ terms are equal. This implies that $\operatorname{Lk}\left(K_{i}, l_{j}^{t}\right)=$ $-\operatorname{Lk}\left(K_{i}^{\prime}, l_{j}^{t}\right)$ for all $1 \leq j \leq \alpha$ and $1 \leq t \leq p$.

Consequently the linking matrix of $L$ is of the form (here coefficients are considered modulo $p$ )

$$
\left(\begin{array}{cccccccc}
0 & 0 & \ldots & 0 & t_{2}^{1} & t_{2}^{2} & \ldots & t_{\alpha}^{p} \\
0 & 0 & \ldots & 0 & -t_{2}^{1} & -t_{2}^{2} & \ldots & -t_{\alpha}^{p} \\
0 & 0 & \ldots & 0 & . & . & \ldots & . \\
0 & 0 & \ldots & 0 & . & . & \ldots & . \\
t_{2}^{1} & -t_{2}^{2} & \ldots & . & . & . & \ldots & . \\
t_{2}^{2} & -t_{2}^{2} & \ldots & . & . & . & \ldots & . \\
. & . & \ldots & . & . & . & \ldots & . \\
. & . & \ldots & . & . & . & \ldots & . \\
. & . & \ldots & . & . & . & \ldots & . \\
t_{\alpha}^{p} & -t_{\alpha}^{p} & \ldots & . & . & . & \ldots & .
\end{array}\right) .
$$

It is well known that the rows of this linking matrix sum to zero. In addition, the first row and the second row of our matrix are dependent. Hence, all the cofactors of the matrix are null. This concludes that $a_{0}$ is null modulo $p$.

Proposition 4.1. If all the links of type $m$ have $a_{2 i-2}$ null modulo $p$, where $2 i<$ $p-1$, then all links of type $m-1$ have $a_{2 i}$ null modulo $p$.

Proof. Assume that for all links $L^{\prime \prime}$ of type $m$, we have $a_{2 i-2}\left(L^{\prime \prime}\right) \equiv 0$ modulo $p$, where $2 i<p-1$. Let $L$ be a link of type $m-1$. By definition we have $L=K_{1} \cup K_{1}^{\prime} \cup \cdots \cup K_{m-1} \cup K_{m-1}^{\prime} \cup L^{\prime}$, where $L^{\prime}$ is a strongly periodic OS link. We start by proving the following lemma.

Lemma 4.4. If $L_{p+}$ and $L_{p-}$ are two p-periodic links of type $m-1$ such that their quotients differ only by a self-crossing change in $\overline{L^{\prime}}$, then $a_{2 i}\left(L_{p+}\right) \equiv a_{2 i}\left(L_{p-}\right)$ modulo $p$.

Proof. If we change a self-crossing in the quotient link $\overline{L^{\prime}}$, then according to Lemma 2.1 , the link $L_{p 0}$ is either of type $m$ having $(\sharp L-p+2)$ components or a periodic link having $(\sharp L+p)$ components. In the second case Remark 4.1 implies that $a_{2 i}\left(L_{p+}\right) \equiv a_{2 i}\left(L_{p-}\right)$ modulo $p$. However in the first case, we get $a_{2 i}\left(L_{p+}\right)-$ $a_{2 i}\left(L_{p-}\right) \equiv a_{2 i-2}\left(L_{p 0}\right)$ modulo $p$. Since $L_{p 0}$ is of type $m$, then $a_{2 i-2}\left(L_{p 0}\right)$ is null modulo $p$. This ends the proof of Lemma 4.4. 
Lemma 4.5. Let $i$ be an integer such that $2 i<p-1$. If $a_{2 i}(L) \equiv 0$ modulo $p$ for links of type $m-1$ with algebraically split orbits, then $a_{2 i}(L) \equiv 0$ for links of type $m-1$.

Proof. The idea of the proof is to use self-crossing changes to split the orbits of our link. The algorithm can be described as follows. We start by changing the crossings of $l_{1}^{1}$ and $l_{1}^{2}$, until their linking number becomes zero. Of course, at the same time we get $l k\left(\varphi_{p}^{i}\left(l_{1}^{1}\right), \varphi_{p}^{i}\left(l_{1}^{2}\right)\right)=0$, for all $1 \leq i \leq p-1$. In the second step we do the same for $l_{1}^{1}$ and $l_{1}^{3}$ and so on. We apply the same procedure for each orbit of components until we get all orbits to be algebraically split.

Return now to the proof of Proposition 4.1. According to Lemma 4.5, it will be enough to prove the proposition in the case where $L$ has only algebraically split orbits. The proof is done by induction on $\alpha$. Remember that the number of components of $L$ is $2(m-1)+p \alpha$. If $\alpha=0$, then $a_{2 i}$ is zero modulo $p$ due to Lemma 4.2.

Now assume that the result is true for links of type $m-1$ with less than $2(m-$ 1) $+p \alpha$ components. Assume that $L$ is a link of type $m-1$ with orbits algebraically split such that $L$ has $2(m-1)+p \alpha$ components.

Since the orbits of $L$ are algebraically split we can use mixed equivariant crossing changes to transform our link into an algebraically split link. According to Lemma 2.1, there are 4 types of mixed equivariant crossing changes:

In the first and the second case of Lemma 2.1, Remark 4.1 implies that $a_{2 i}\left(L_{p+}\right)-$ $a_{2 i}\left(L_{p-}\right) \equiv 0$ modulo $p$.

In the third and the fourth case, Lemma 4.1 implies that $a_{2 i}\left(L_{p+}\right)-a_{2 i}\left(L_{p-}\right) \equiv$ $a_{2 i}\left(L_{p 0}\right)$ modulo $p$. Since the link $L_{p 0}$ is of type $m-1$ having fewer components than the original link $L$, we can use the induction hypothesis to conclude that $a_{2 i}\left(L_{p+}\right)-a_{2 i}\left(L_{p-}\right) \equiv 0$ modulo $p$.

In conclusion, mixed equivariant crossing changes do not affect the coefficient $a_{2 i}$ modulo $p$. Using these changes we should be able to transform our link into an algebraically split link having $a_{2 i}$ null modulo $p$. This completes the proof of Proposition 4.1.

The rest of the proof of the Main Theorem is a matter of induction. According to Lemma 4.3, a link of type $(p-1) / 2$ has $a_{0}$ null modulo $p$. Starting from this fact, an easy induction using Proposition 4.1 shows that a link of type zero (which is a strongly periodic OS link) has $a_{2 i}$ null modulo $p$ for all $i$ such that $2 i<p-1$. This completes the proof of the Main Theorem.

\section{REFERENCES}

[1] H. Bass and J. W. Morgan. The Smith conjecture. Pure and Appl. Math. 112, Academic Press, Orlando, FL (1984). MR0758459 (86i:57002)

[2] G. Burde. Über periodische Knoten. Arch. Math. (Basel) 30 (1978), 487-492. MR0645216 $(58: 31051)$

[3] N. ChBILI. Les invariants $\theta_{p}$ des 3-variétés périodiques. Annales de l'Institut Fourier (Grenoble) 51, Fascicule 4 (2001), 1135-1150. MR1849218 (2002e:57014)

[4] N. ChBILI. Quantum invariants and finite group actions on 3-manifolds, Topology Appl. 136/1-3 (2004), 219-231. MR2023419 (2004k:57015)

[5] N. ChBiLi. The Casson-Walker-Lescop invariant of periodic three-manifolds, Math. Proc. Cambridge Philos. Soc. 140, 2 (2006), 253-264. MR.2212278 (2006k:57033)

[6] Q. Chen and T. Le. Quantum invariants of periodic links and periodic 3-manifolds. Fund. Math. 184 (2004), 55-71. MR2128042 (2005k:57022) 
[7] J. F. Davis and C. Livingston. Alexander polynomials of periodic knots. Topology 30 (1991), 551-564. MR:1133872 (92k:57008)

[8] P. Gilmer, J. Kania-Bartoszynska, and J. Przytycki. 3-Manifold invariants and periodicity of homology spheres. Algebraic and Geometric Topology 2 (2002), 825-842. MR1936972 (2004f:57019)

[9] R. Hartley. The Conway potential function for links. Comment. Math. Helv. 58 (1983), no. 3, 365-378. MR727708 (85h:57006)

[10] J. Hillman. New proofs of two theorems on periodic knots. Arch. Math. (Basel) 37 (1981), 457-461. MR643289 (83b:57003)

[11] F. HosokawA. On $\nabla$-polynomials of links. Osaka Math. J. 10 (1958), 273-282. MR0102820 $(21: 1606)$

[12] J. Hoste. The first coefficient of the Conway polynomial. Proc. Amer. Math. Soc. 95 (1985), 299-302. MR801342 (86m:57009)

[13] C. Lescop. Global surgery formula for the Casson-Walker invariant, Annals of Mathematics Studies 140, Princeton Univ. Press (1996). MR1372947 (97c:57017)

[14] J. Levine. The Conway polynomial of an algebraically split link. Proceedings of Knots '96 (Tokyo), edited by S. Suzuki, World Scientific Publishing Co., River Edge, NJ (1997), pp. 2329. MR 1664948 (99j:57008)

[15] Y. MiYaZAWA. Conway polynomials of periodic links. Osaka J. Math. 31 (1994), 147-163. MR.1262794 (95e:57018)

[16] K. Murasugi. On periodic knots. Comment. Math. Helv. 46 (1971), 162-174. MR0292060 $(45: 1148)$

[17] J. H. PrZYtycki. On Murasugi's and Traczyk's criteria for periodic links. Math. Ann. 283 (1989), 465-478. MR985242(90e:57015)

[18] J. PRZYTYCKI and M. SokOLOV. Surgeries on periodic links and homology of periodic 3-manifolds. Math. Proc. Cambridge Phil. Soc. 131(2) (2001), 295-307. MR1857121 (2002g:57017)

[19] M. SaKuma. On the polynomials of periodic links. Math. Ann. 257 (1981), 487-494. MR639581 (83i:57003)

[20] M. SAKUMA. Surgery description of orientation-preserving periodic maps on compact orientable 3-manifolds. Rend. Istit. Mat. Univ. Trieste 32 (2001), suppl. 1, 375-396 (2002). MR.1893406 (2003d:57043)

Osaka City University Advanced Mathematical Institute, Sugimoto 3-3-138, SumiYoshi-Ku 5588585 OSAKA, JAPAN

E-mail address: chbili@sci.osaka-cu.ac.jp 